Kemal Idrizovic ${ }^{1}$

Natasa Zenic ${ }^{2}$

Enver Tahiraj

Nikola Rausavljevic ${ }^{2}$

Damir Sekulic ${ }^{2,4}$

\title{
CIGARETTE SMOKING AMONG 17-18 YEAR OLD ADOLESCENTS - PREVALENCE AND ASSOCIATION WITH SOCIODEMOGRAPHIC, FAMILIAL, SPORT, AND SCHOLASTIC FACTORS
}

\author{
PALENIE PAPIEROSÓW WŚRÓD MŁODZIEŻY W WIEKU 17-18 LAT - SKALA ZJAWISKA \\ I JEGO ZWIAZZEK Z CZYNNIKAMI SOCJODEMOGRAFICZNYMI, RODZINNYMI, \\ UPRAWIANIEM SPORTU I POSTĘPAMI W NAUCE
}

\footnotetext{
${ }^{1}$ University of Podgorica, Podgorica, Monte Negro

Faculty of Sport and Physical Education

${ }^{2}$ University of Split, Split, Croatia

Faculty of Kinesiology

${ }^{3}$ Ministry of Culture, Pristina, Republic of Kosovo

Youth and Sports of Republic of Kosovo

${ }^{4}$ University of Split, Split, Croatia

University Department of Health Care Studies
}

\begin{abstract}
Background: Though adolescence is recognised as a critical period for smoking prevention, there is a lack of research focused on this issue in Kosovo. The aim of this study has been to examine the gender-specific factors of influence (predictors) for smoking among adolescents in Pristina, Kosovo. Material and Methods: The study sample comprised 1002 adolescents at the age of 17-18 (366 boys, 636 girls), all of whom were in the school's 12th grade. The predictors included sociodemographic variables, familial (i.e., parental) monitoring, parents' educational background, and sport-related factors. The $\mathrm{Chi}^{2}$ and forward stepwise logistic regression analyses with a dichotomous criterion (smoking vs. non-smoking) were applied. Results: The incidence of smoking was high (31\% and $40 \%$ smokers, including $7 \%$ and $12 \%$ daily smokers for girls and boys, respectively). The regression model revealed more frequent absence from school (odds ratio (OR): 1.544; 95\% confidence interval (CI): 1.063-2.243), more unexcused school absences (OR: 1.360; 95\% CI: 1.029-1.796), and frequent parental questioning (OR: 1.530; 95\% CI: 1.020-2.295) to be significant predictors of smoking among boys. For girls, a higher risk of smoking was associated with lower scholastic achievement (OR: 1.467; 95\% CI: 1.089-1.977), more frequent absence from school (OR: 1.565; 95\% CI: 1.137-2.155), increased conflict with parents (OR: 1.979; 95\% CI: 1.405-2.789), and a self-declared perception of less parental care (OR: 0.602; 95\% CI: 0.377-0.962). Sports were not found to be strongly related to smoking. However, a high risk of daily smoking was found among boys who participated in team sports and subsequently quit. Conclusions: This study reinforces the need for gender- and culture-specific approaches to studying the factors that influence smoking among adolescents. Med Pr 2015;66(2):153-163
\end{abstract}

Key words: predictors, logistic regression, substance, physical exercising, cigarette smoking, adolescents

\section{STRESZCZENIE}

Wstęp: Mimo że wiek dorastania jest uważany za okres bardzo ważny z punktu widzenia zapobiegania nałogowi palenia tytoniu, nie ma badań poświęconych temu tematowi w Kosowie. Celem niniejszej pracy było zbadanie specyficznych dla płci czynników skłaniających do palenia młodzież z Prisztiny w Kosowie. Materiał i metody: Badaniami objęto 1002 osoby w wieku 17-18 lat (366 chłopców, 636 dziewcząt) z 12. klasy. Pod uwagę wzięto zmienne socjodemograficzne, nadzór rodzicielski, poziom wykształcenia rodziców i czynniki związane z uprawianiem sportu. Zastosowano test $\mathrm{Chi}^{2}$ oraz analizę regresji logistycznej metoda krokową z zastosowaniem zmiennej kryterialnej (palenie vs niepalenie). Wyniki: Odsetek palaczy był wysoki (papierosy paliło $40 \%$ chłopców i $31 \%$ dziewcząt, w tym 7\% dziewcząt i $12 \%$ chłopców codziennie). Model regresji wykazał, że częstsza nieobecność w szkole (iloraz szans (odds ratio - OR): 1,544; 95\% przedział ufności (confidence interval - CI): 1,063-2,243), więcej nieusprawiedliwionych nieobecności w szkole (OR: 1,360; 95\% CI: 1,029-1,796) i nadmierne zainteresowanie rodziców (OR: 1,530; 95\% CI: 1,020-2,295) były istotnymi predyktorami palenia wśród chłopców. Wśród dziewcząt wyższe ryzyko palenia wynikało z gorszych postępów w nauce (OR: 1,467; 95\% CI: 1,089-1,977), częstszej nieobecności w szkole (OR: 1,565; 95\% CI: 1,137-2,155), częstszych konfliktów z rodzicami (OR: 1,979; 95\% CI: 1,405-2,789) i poczucia zbyt małej opieki rodzicielskiej (OR: 0,602; 95\% CI: 0,377-0,962). Okazało się, 
że uprawianie sportu nie ma istotnego wpływu na nałóg palenia. Stwierdzono jednak wysokie ryzyko codziennego palenia przez chłopców, którzy przestali uprawiać sport zespołowy. Wnioski: Wyniki niniejszej pracy potwierdzają, że badając czynniki, które wpływają na palenie tytoniu wśród młodzieży, należy brać pod uwagę różnice związane z płcią i kulturą. Med. Pr. 2015;66(2):153-163

Słowa kluczowe: czynniki prognostyczne, regresja logistyczna, substancja, ćwiczenia fizyczne, palenie papierosów, młodzież

Corresponding author / Autor do korespondencji: Damir Sekulic, University of Split, Teslina 6, Split 21000, Croatia, e-mail: dado@kifst.hr

Received: September 29, 2014, accepted: November 21, 2014

\section{INTRODUCTION}

Despite the decrease in prevalence globally, smoking remains the most common type of substance misuse in the world [1]. Statistics and national data on substance use and abuse in the territory of Kosovo are scarce but reported data shows that the prevalence of smoking is high. To the best of our knowledge, only 1 study has investigated the problem of substance misuse, and the authors have reported that $36 \%$ of adolescents in the city of Gjilan were smokers, with no significant difference between girls and boys [2]. These findings are consistent with previous studies on the prevalence of smoking among adolescents in the region (a former Yugoslav territory) $[3,4]$.

Identifying protective and risk factors related to smoking will allow public health authorities to define specific and targeted preventive campaigns to emphasise and encourage the protective factors and to control the risk factors $[4,5]$. Since cigarette smoking is viewed to be addiction, it is important to identify the factors associated with cigarette use.

Although strongly proclaimed as protective, sports (i.e., physical exercise) have not been consistently validated as a buffering factor against cigarette smoking among adolescents [6]. While some studies have indicated that sports and physical activity are protective, other studies have shown an increased likelihood of smoking among individuals who practise sports $[4,7,8]$. Another factor worth studying is religiosity and its influence on substance misuse [9]. However, the relationship between substance misuse and religiosity/spirituality among adolescents is not clear. Accordingly, studies have found that the influence of different religions on the consumption of substances is variable [10-12]. Parental factors and parental (i.e., familial) monitoring have generally been found to be protective against cigarette smoking, and studies have been consistent in this regard irrespective of differences in the study populations $[13,14]$.

No recent study has systematically investigated the factors related to cigarette smoking among adolescents in Kosovo. Additionally, although frequently studied separately, familial factors (i.e., parents' educational background and familial monitoring), religiosity, and sports participation have not been extensively studied together to determine their potential association with cigarette smoking among adolescents.

The aim of this investigation has been to explore the gender-specific relationships between sociodemographic variables, parental monitoring, scholastic achievement and sports-related factors as predictors of daily cigarette smoking among adolescents in Pristina, Kosovo. Given that previous studies have indicated that attitudes toward substance misuse vary among religions and ethnicities, and to prevent the possible confounding effects of these variations, we have limited our sample to adolescents who declared themselves as ethnic Albanians and traditionally Islamic regardless of their current strength of religious faith. The main hypothesis of this work is that the observed sociodemographic, familial monitoring, sport, and educational factors are independently associated with cigarette smoking prevalence among adolescents from Pristina, Kosovo.

\section{MATERIAL AND METHODS}

\section{Subjects}

This study enrolled 1002 adolescents - 17- and 18-yearolds ( 366 boys, 636 girls). All subjects were the last school year's students within the framework of the compulsory education (12th grade). The sample represents $37 \%$ of the high school seniors in Pristina, which is approximately $5 \%$ of the high school seniors in Kosovo. The sample was intentionally limited to 17 - and 18-year-old children because studies conducted to date in the former Yugoslav territories have studied this age group, thus allowing us to objectively compare findings [2-4].

\section{Variables}

Surveys were performed using a self-administered questionnaire, which was previously used and found to 
be valid in a similar sample of subjects in surrounding countries $[3,4]$. Although the questionnaire consisted of a broad variety of questions, the study focused on sociodemographic variables, sport-related factors, familial monitoring (predictors), and cigarette consumption data (dependent variable).

The following sociodemographic data was collected: age, gender, ethnicity, self-declared religion, and religiosity, as measured by the Santa Clara Strength of Religious Faith Questionnaire (SCSRF). Additionally, subjects reported the educational background of their parents (separately for mother and father using a 4-point scale ranging from "elementary school" to "university degree") and their estimated family income (a 3-point scale that included "below average," "average," and "above average").

Familial monitoring was assessed using the following 4 questions:

1. "How often do you have a conflict with your parents?"

2. "How often are your parents absent from home, including for their job obligations?"

3. "How often do your parents ask you questions about your friends, scholastic achievement, problems, and other personal issues?" (the subjects responded on a 4-point scale ranging from "almost never" to "regularly").

4. "How would you rate how much your parents care about you and your personal life?" (the subjects responded on a 4-point scale that included "They do not care at all," "They do not care enough," "They are relatively concerned," and "They are highly concerned").

We assessed participation in individual and team sports separately (both assessments used 3-point scales that included the responses "Yes, I'm still participating," "Yes, but not anymore," and "No, never"). We also assessed the amount of time subjects spent engaging in sports (using a 4-point scale ranging from "Never involved in sports" to " $5+$ years") and their competitive sports achievements (using a 4-point scale ranging from "Never competed" to "National team").

Smoking was assessed on a 6-point scale ("I have never smoked," "Quit smoking," "Occasionally, from time to time, but not daily," "Fewer than 10 cigarettes per day," "10-20 cigarettes per day," and "More than a pack daily").

\section{Surveys and ethics}

In the 1st phase of our sampling procedure, we randomly selected a half of the schools in the territory of
Pristina, the capital of Kosovo. In the next phase, 1/3rd of all 12 th grade classes were randomly selected (i.e., 31 classes from the previously sampled schools). The survey was administered on a single day, meaning that all 17- and 18-year-old children who were at school on that day were included in the study. Before administering the survey, we explained the full procedure and study aims to all subjects and to at least 1 parent per subject, and an informed consent was obtained. In this study, we included only those subjects who identified themselves as traditionally Islamic (innate religious tendency), regardless of their SCSRF score, as explained previously.

The survey was strictly anonymous, meaning that we tried not to ask for personal data (e.g., birth date, the city of birth, a club or sports participation). Multiplechoice answers were offered when possible. The survey was administered for groups of at least 15 respondents. Each respondent was informed that the survey was strictly anonymous, that they could refuse to participate, and that they could leave questions unanswered. For the purpose of reliable analyses, the examinees were asked to use a confidential code, which was not known to anyone but the examinees themselves, for identification purposes.

Each respondent received 2 questionnaire forms and 1 envelope. After completing the survey, the subjects placed 1 form (answered or unanswered) in the envelope. The second form was shredded in a paper shredder. The response rate was over $99 \%$. To verify the reliability of the questionnaire, we randomly selected 42 examinees to be tested twice within a 2-week period. The study fulfilled all ethical guidelines and received the approval of the authors' institutional ethics board.

\section{Statistics}

All variables except for the SCSRF score were identified as nonparametric (i.e., qualitative); therefore, we calculated counts and frequencies as descriptive statistics. The reliability of the questionnaire was evaluated using the test-retest Pearson correlation for the SCSRF score and the Spearman rank correlation for ordinal variables. Additionally, nonparametric variables were assessed in terms of reliability by calculating the percentage share of the equally responded queries. This is a common procedure in defining the consistency of measurement for questionnaires and investigations using surveys $[15,16]$. Gender differences were evaluated using the Kruskal-Wallis Anova (KW) for all predictors except for the SCSRF score, the Chi ${ }^{2}$ test - for cigarette smoking, and the Student's t-test - for the SCSRF score. The Chi ${ }^{2}$ 
test was applied with a dichotomous dependent criterion (daily smoking) to identify the univariate association between each predictor and smoking.

The gender-specific relationships between the predictors and the criterion were examined using forward conditional logistic regression calculations. More precisely, we performed 2 multiple logistic regressions ( 1 for each gender) to predict the categorical criterion of daily cigarette smoking ("0" for "I have never smoked," "Quit smoking" and "Occasionally, from time to time, but not daily" and " 1 " for the remaining responses). The predictors were sociodemographic variables, scholastic variables, sport-related factors, and parental/familial monitoring variables. A forward stepwise conditional regression model using assuming 95\% of statistical significance was applied.

\section{RESULTS}

The reliability of the applied questionnaire was high. The test-retest Pearson correlation for the SCSRF score was $0.91(\mathrm{p}<0.05)$, and the Spearman rank order correlation for ordinal variables ranged from 0.85 for sociodemographic variables to 0.98 for scholastic variables (all significant at $\mathrm{p}<0.05$ ).

Boys smoked cigarettes more than girls did (KW test $=8.38 ; \mathrm{p}<0.01$ ). Almost $40 \%$ of boys and $31 \%$ of girls were smokers, while $12 \%$ of boys and $7 \%$ of girls reported daily smoking (Table 1).

Girls reported significantly better educational achievements. A significant association was found between school absences and daily smoking (smoking was more frequent among those who were more frequently absent

Table 1. Smoking incidence in the study group

Tabela 1. Częstość palenia w badanej grupie

\begin{tabular}{lrrrr}
\hline \multicolumn{1}{c}{$\begin{array}{c}\text { Smoking } \\
\text { Palenie papierosów }\end{array}$} & $\begin{array}{c}\text { Girls } \\
\text { Dziewczęta } \\
(\mathrm{N}=636)\end{array}$ & \multicolumn{2}{c}{$\begin{array}{c}\text { Boys } \\
\text { Chłopcy } \\
(\mathrm{N}=366)\end{array}$} \\
\cline { 2 - 6 } & $\mathrm{n}$ & $\%$ & $\mathrm{n}$ & $\%$ \\
\hline $\begin{array}{c}\text { I have never smoked / Nigdy nie } \\
\text { paliłam/em }\end{array}$ & 428 & 67.3 & 206 & 56.3 \\
Quit smoking / Przestałam/em palić & 20 & 3.1 & 14 & 3.8 \\
Occasionally, from time to time / & 145 & 22.8 & 99 & 27.0 \\
Okazjonalnie, od czasu do czasu & & & & \\
Every day / Codziennie & & & & \\
$\quad<10$ cigarettes / papierosów & 26 & 4.1 & 19 & 5.2 \\
10-20 cigarettes / papierosów & 15 & 2.4 & 26 & 7.1 \\
$>20$ cigarettes / papierosów & 2 & 0.3 & 2 & 0.5 \\
\hline
\end{tabular}

from school), unexcused school absences and smoking (smoking was more frequent for those students who had more unexcused absences) (Table 2).

Boys dominated in the case of all sport-related factors that were studied. A univariate association between smoking and participation in team sports was found for boys, with the highest incidence among those boys who quit team sports (Table 3 ).

No significant difference between genders was observed in familial financial status or parental education (Table 4).

Students' reports of parental questioning (i.e., parents' interest in their children's friends, school, and other personal issues), overall parental care, and conflict with parents were significantly higher among girls. Boys and girls who reported themselves to be in a higher conflict with parents were more likely to be smokers (Table 5).

Although high levels of religiosity were reported, there was no significant gender difference in the SCSRF scores $(35.3 \pm 7.7$ and $36.5 \pm 6.3$ for boys and girls, respectively; $\mathrm{t}$-value $=-1.54 ; \mathrm{p}>0.05)$.

The regression models were successful in obtaining the predictive values for both boys and girls. The scholastic variables were the most significant predictors of daily smoking. Among boys, more frequent absences from school (odds ratio (OR): 1.544; 95\% confidence interval (CI): 1.063-2.243); more unexcused school absences (OR: 1.360; 95\% CI: 1.029-1.796); and frequent parental questioning (OR: 1.530; 95\% CI: 1.020-2.295) were included in the successful regression model.

For girls, a higher risk of daily smoking was associated with lower scholastic achievement (OR: 1.467; 95\% CI: 1.089-1.977), more frequent absences from school (OR: 1.565; 95\% CI: 1.137-2.155), increased conflict with parents (OR: 1.979; 95\% CI: 1.405-2.789), and a self-reported perception of less parental/familial care (OR: 0.602; 95\% CI: 0.377-0.962) (Table 6). In the final step (the 3rd one for boys and the 4th one for girls), the logistic regressions successfully classified $62 \%$ of boys ( $83 \%$ non-smokers and $50 \%$ smokers) and $72 \%$ of girls (91\% non-smokers and 33\% smokers).

\section{DISCUSSION}

This study has come forward with several important findings. Firstly, the descriptive data shows a rate of cigarette smoking among adolescents that is consistent with the literature. Secondly, the studied variables have had a similar association with cigarette smoking in the case of boys and girls. Thirdly, the study has confirmed 
Table 2. Scholastic variables - univariate association between incidence of smoking and studied variables

Tabela 2. Zmienne związane ze szkołą - jednoczynnikowa zależność między częstością palenia a badanymi zmiennymi

\begin{tabular}{|c|c|c|c|c|c|c|c|}
\hline \multirow[t]{2}{*}{$\begin{array}{l}\text { Variable } \\
\text { Zmienna }\end{array}$} & \multicolumn{2}{|c|}{$\begin{array}{c}\text { Girls } \\
\text { Dziewczęta } \\
(\mathrm{N}=636) \\
{[\mathrm{n}(\%)]}\end{array}$} & \multirow[t]{2}{*}{$\begin{array}{c}\mathrm{p} \\
\left(\mathrm{Chi}^{2}\right)\end{array}$} & \multicolumn{2}{|c|}{$\begin{array}{c}\text { Boys } \\
\text { Chłopcy } \\
(\mathrm{N}=366) \\
{[\mathrm{n}(\%)]}\end{array}$} & \multicolumn{2}{|c|}{$\mathrm{p}$} \\
\hline & $\begin{array}{l}\text { total } \\
\text { ogółem }\end{array}$ & $\begin{array}{l}\text { smokers } \\
\text { palacze }\end{array}$ & & $\begin{array}{c}\text { total } \\
\text { ogółem }\end{array}$ & $\begin{array}{l}\text { smokers } \\
\text { palacze }\end{array}$ & $\mathrm{Chi}^{2}$ & KW \\
\hline Scholastic achievement / Postępy w nauce & & & & & & * & 1.00 \\
\hline excellent / wspaniałe & $376(59.12)$ & $96(47.52)$ & & $132(36.07)$ & $52(34.21)$ & & \\
\hline very good / bardzo dobre & $146(22.96)$ & $48(23.76)$ & & $146(39.89)$ & $62(40.79)$ & & \\
\hline good - average / dobre - średnie & $94(14.78)$ & $40(19.80)$ & & $82(22.40)$ & $36(23.68)$ & & \\
\hline sufficient - below average / wystarczające - poniżej średniej & $18(2.83)$ & $16(7.92)$ & & $4(1.090)$ & $2(1.32)$ & & \\
\hline insufficient / niewystarczające & $2(0.31)$ & $2(0.99)$ & 0.10 & $2(0.55)$ & $0(0.00)$ & & \\
\hline School absence / Nieobecność w szkole & & & & & & 0.01 & 0.40 \\
\hline almost never / prawie nigdy & $138(21.70)$ & $22(10.89)$ & & $88(24.04)$ & $24(15.79)$ & & \\
\hline rarely / rzadko & $218(34.28)$ & $54(26.73)$ & & $108(29.51)$ & $30(19.74)$ & & \\
\hline from time to time / od czasu do czasu & $240(37.74)$ & $102(50.50)$ & & $114(31.15)$ & $60(39.47)$ & & \\
\hline often / często & $40(6.29)$ & $24(11.88)$ & 0.01 & $54(14.75)$ & $38(25.00)$ & & \\
\hline $\begin{array}{l}\text { Unexcused school absence / Nieusprawiedliwiona } \\
\text { nieobecność w szkole }\end{array}$ & & & & & & 0.01 & 0.01 \\
\hline$<5$ & $260(40.88)$ & $48(23.76)$ & & $88(24.04)$ & $20(13.16)$ & & \\
\hline $5-10$ & $184(28.93)$ & $62(30.69)$ & & $104(28.42)$ & $38(25.00)$ & & \\
\hline $11-15$ & $88(13.84)$ & $38(18.81)$ & & $80(21.86)$ & $34(22.37)$ & & \\
\hline $16-20$ & $44(6.92)$ & $14(6.93)$ & & $38(10.38)$ & $20(13.16)$ & & \\
\hline$>20$ & $58(9.12)$ & $38(18.81)$ & 0.01 & $56(15.30)$ & $40(26.32)$ & & \\
\hline Behavior grade / Zachowanie & & & & & & 0.45 & 0.01 \\
\hline exemplary / wzorowe & $518(81.45)$ & $146(72.28)$ & & $258(70.49)$ & $98(64.47)$ & & \\
\hline fair / dobre & $100(15.72)$ & $50(24.75)$ & & $100(27.32)$ & $50(32.89)$ & & \\
\hline poor / nieodpowiednie & $16(2.52)$ & $6(2.97)$ & 0.10 & $8(2.19)$ & $4(2.63)$ & & \\
\hline
\end{tabular}

KW - Kruskal-Wallis test / test Kruskala-Wallisa.

${ }^{*} \mathrm{Chi}^{2}$ was not calculated because of the null frequency / Chi ${ }^{2}$ nie wyznaczano z powodu zerowej frekwencji.

Table 3. Sport factors - univariate association between incidence of smoking and studied variables

Tabela 3. Czynniki związane z uprawianiem sportu - jednoczynnikowa zależność między częstością palenia a badanymi zmiennymi

\begin{tabular}{|c|c|c|c|c|c|c|c|}
\hline \multirow[t]{2}{*}{$\begin{array}{l}\text { Variable } \\
\text { Zmienna }\end{array}$} & \multicolumn{2}{|c|}{$\begin{array}{c}\text { Girls } \\
\text { Dziewczęta } \\
(\mathrm{N}=636) \\
{[\mathrm{n}(\%)]}\end{array}$} & \multirow[t]{2}{*}{$\underset{\left(\mathrm{Chi}^{2}\right)}{\mathrm{p}}$} & \multicolumn{2}{|c|}{$\begin{array}{c}\text { Boys } \\
\text { Chłopcy } \\
(\mathrm{N}=366) \\
{[\mathrm{n}(\%)]}\end{array}$} & \multicolumn{2}{|c|}{$\mathrm{p}$} \\
\hline & $\begin{array}{c}\text { total } \\
\text { ogółem }\end{array}$ & $\begin{array}{l}\text { smokers } \\
\text { palacze }\end{array}$ & & $\begin{array}{c}\text { total } \\
\text { ogółem }\end{array}$ & $\begin{array}{l}\text { smokers } \\
\text { palacze }\end{array}$ & $\mathrm{Chi}^{2}$ & KW \\
\hline \multicolumn{8}{|l|}{$\begin{array}{l}\text { Participation in individual sports / Uprawianie } \\
\text { indywidualnych dyscyplin sportowych }\end{array}$} \\
\hline yes, I'm still participating / tak, uprawiam nadal & $148(23.27)$ & $58(28.71)$ & & $148(40.44)$ & $58(38.16)$ & & \\
\hline yes, but not anymore / kiedyś tak, teraz nie & $196(30.82)$ & $56(27.72)$ & & $118(32.24)$ & $58(38.16)$ & & \\
\hline no, never / nie, nigdy & $292(45.91)$ & $88(43.56)$ & 0.47 & $100(27.32)$ & $36(23.68)$ & 0.45 & 0.01 \\
\hline \multicolumn{8}{|l|}{$\begin{array}{l}\text { Participation in team sports / Uprawianie zespołowych } \\
\text { dyscyplin sportowych }\end{array}$} \\
\hline yes, I'm still participating / tak, uprawiam stale & $132(20.75)$ & $48(23.76)$ & & $146(39.89)$ & $60(39.47)$ & & \\
\hline yes, but not anymore / kiedyś tak, teraz nie & $180(28.30)$ & $54(26.73)$ & & $124(33.88)$ & $64(42.11)$ & & \\
\hline no, never / nie, nigdy & $324(50.94)$ & $100(49.50)$ & 0.77 & $94(25.68)$ & $26(17.11)$ & 0.04 & 0.01 \\
\hline
\end{tabular}


Table 3. Sport factors - univariate association between incidence of smoking and studied variables - cont.

Tabela 3. Czynniki związane z uprawianiem sportu - jednoczynnikowa zależność między częstością palenia a badanymi zmiennymi - cd.

\begin{tabular}{|c|c|c|c|c|c|c|c|}
\hline \multirow[t]{2}{*}{$\begin{array}{l}\text { Variable } \\
\text { Zmienna }\end{array}$} & \multicolumn{2}{|c|}{$\begin{array}{c}\text { Girls } \\
\text { Dziewczęta } \\
(\mathrm{N}=636) \\
{[\mathrm{n}(\%)]}\end{array}$} & \multirow[t]{2}{*}{$\underset{\left(\mathrm{Chi}^{2}\right)}{\mathrm{p}}$} & \multicolumn{2}{|c|}{$\begin{array}{c}\text { Boys } \\
\text { Chłopcy } \\
(\mathrm{N}=366) \\
{[\mathrm{n}(\%)]}\end{array}$} & \multicolumn{2}{|c|}{$\mathrm{p}$} \\
\hline & $\begin{array}{l}\text { total } \\
\text { ogółem }\end{array}$ & $\begin{array}{l}\text { smokers } \\
\text { palacze }\end{array}$ & & $\begin{array}{c}\text { total } \\
\text { ogółem }\end{array}$ & $\begin{array}{l}\text { smokers } \\
\text { palacze }\end{array}$ & $\mathrm{Chi}^{2}$ & KW \\
\hline \multicolumn{8}{|l|}{$\begin{array}{l}\text { Period of the sports involvement / Okres uprawiania } \\
\text { sportu }\end{array}$} \\
\hline never involved / nigdy & $244(38.36)$ & $78(38.61)$ & & $54(14.75)$ & $12(7.89)$ & & \\
\hline$<1$ year / rok & $214(33.65)$ & $56(27.72)$ & & $86(23.50)$ & $36(23.68)$ & & \\
\hline $1-5$ years / lat & $122(19.18)$ & $48(23.76)$ & & $120(32.79)$ & $54(35.53)$ & & \\
\hline$>5$ years / lat & $56(8.81)$ & $20(9.90)$ & 0.52 & $104(28.42)$ & $48(31.58)$ & 0.09 & 0.01 \\
\hline \multicolumn{8}{|l|}{$\begin{array}{l}\text { Competitive achievement in sport / Uczestnictwo } \\
\text { w zawodach sportowych }\end{array}$} \\
\hline never competed / nigdy & $544(85.53)$ & $174(86.14)$ & & $212(57.92)$ & $86(56.58)$ & & \\
\hline $\begin{array}{l}\text { competition of the lower ranks / zawody niższego } \\
\text { szczebla }\end{array}$ & $70(11.01)$ & $24(11.88)$ & & $78(21.31)$ & $38(25.00)$ & & \\
\hline $\begin{array}{l}\text { competition of national level / zawody na szczeblu } \\
\text { krajowym }\end{array}$ & $16(2.52)$ & $4(1.98)$ & & $54(14.75)$ & $24(15.79)$ & & \\
\hline national team / reprezentacja kraju & $6(0.94)$ & $0(0.00)$ & * & $22(6.01)$ & $4(2.63)$ & 0.17 & 0.01 \\
\hline
\end{tabular}

Abbreviations as in Table 2 / Skróty jak w tabeli 2.

Table 4. Familial (parental) status - univariate association between incidence of smoking and studied variables

Tabela 4. Status rodzinny - jednoczynnikowa zależność między częstością palenia a badanymi zmiennymi

\begin{tabular}{|c|c|c|c|c|c|c|c|}
\hline \multirow{2}{*}{$\begin{array}{l}\text { Variable } \\
\text { Zmienna }\end{array}$} & \multicolumn{2}{|c|}{$\begin{array}{c}\text { Girls } \\
\text { Dziewczęta } \\
(\mathrm{N}=636) \\
{[\mathrm{n}(\%)]}\end{array}$} & \multirow{2}{*}{$\begin{array}{c}\mathrm{p} \\
\left(\mathrm{Chi}^{2}\right)\end{array}$} & \multicolumn{2}{|c|}{$\begin{array}{c}\text { Boys } \\
\text { Chłopcy } \\
(\mathrm{N}=366) \\
{[\mathrm{n}(\%)]}\end{array}$} & \multicolumn{2}{|c|}{$\mathrm{p}$} \\
\hline & $\begin{array}{c}\text { total } \\
\text { ogółem }\end{array}$ & $\begin{array}{l}\text { smokers } \\
\text { palacze }\end{array}$ & & $\begin{array}{l}\text { total } \\
\text { ogółem }\end{array}$ & $\begin{array}{l}\text { smokers } \\
\text { palacze }\end{array}$ & $\mathrm{Chi}^{2}$ & KW \\
\hline \multicolumn{8}{|l|}{ Financial status / Stan majątkowy } \\
\hline below average / poniżej przeciętnego & $8(1.26)$ & $2(0.99)$ & & $8(2.19)$ & $4(2.63)$ & & \\
\hline average / przeciętny & $530(83.33)$ & $162(80.20)$ & & $298(81.42)$ & $124(81.58)$ & & \\
\hline above average / powyżej przeciętnego & $94(14.78)$ & $36(17.82)$ & 0.70 & $60(16.39)$ & $24(15.79)$ & 0.95 & 0.99 \\
\hline \multicolumn{8}{|l|}{ Father's education / Wykształcenie ojca } \\
\hline elementary / podstawowe & $12(1.89)$ & $2(0.99)$ & & $6(1.64)$ & $4(2.63)$ & & \\
\hline high school / gimnazjalne & $162(25.47)$ & $46(22.77)$ & & $86(23.50)$ & $36(23.68)$ & & \\
\hline college degree / licealne & $216(33.96)$ & $70(34.65)$ & & $100(27.32)$ & $28(18.42)$ & & \\
\hline university / studia wyższe & $246(38.68)$ & $84(41.58)$ & 0.72 & $174(47.54)$ & $84(55.26)$ & 0.12 & 0.10 \\
\hline \multicolumn{8}{|l|}{ Mother's education / Wykształcenie matki } \\
\hline elementary / podstawowe & $88(13.84)$ & $24(11.88)$ & & $36(9.84)$ & $16(10.53)$ & & \\
\hline high school / gimnazjalne & $240(37.74)$ & $56(27.72)$ & & $126(34.43)$ & $48(31.58)$ & & \\
\hline college degree / licealne & $148(23.27)$ & $58(28.71)$ & & $92(25.14)$ & $26(17.11)$ & & \\
\hline university / studia wyższe & $160(25.16)$ & $64(31.68)$ & 0.10 & $112(30.60)$ & $62(40.79)$ & 0.09 & 0.01 \\
\hline
\end{tabular}

Abbreviations as in Table 2 / Skróty jak w tabeli 2. 
Table 5. Familial (parental) monitoring - univariate association between incidence of smoking and studied variables Tabela 5. Nadzór rodzicielski - jednoczynnikowa zależność między częstością palenia a badanymi zmiennymi

\begin{tabular}{|c|c|c|c|c|c|c|c|}
\hline \multirow[t]{2}{*}{$\begin{array}{l}\text { Variable } \\
\text { Zmienna }\end{array}$} & \multicolumn{2}{|c|}{$\begin{array}{c}\text { Girls } \\
\text { Dziewczęta } \\
(\mathrm{N}=636) \\
{[\mathrm{n}(\%)]}\end{array}$} & \multirow[t]{2}{*}{$\underset{\left(\mathrm{Chi}^{2}\right)}{\mathrm{p}}$} & \multicolumn{2}{|c|}{$\begin{array}{c}\text { Boys } \\
\text { Chłopcy } \\
(\mathrm{N}=366) \\
{[\mathrm{n}(\%)]}\end{array}$} & \multicolumn{2}{|c|}{$\mathrm{p}$} \\
\hline & $\begin{array}{l}\text { total } \\
\text { ogółem }\end{array}$ & $\begin{array}{l}\text { smokers } \\
\text { palacze }\end{array}$ & & $\begin{array}{l}\text { total } \\
\text { ogółem }\end{array}$ & $\begin{array}{l}\text { smokers } \\
\text { palacze }\end{array}$ & $\mathrm{Chi}^{2}$ & KW \\
\hline \multicolumn{8}{|l|}{ Familial conflicts / Konflikty z rodzicami } \\
\hline almost never / prawie nigdy & $100(15.72)$ & $14(6.93)$ & & $98(26.78)$ & $24(15.79)$ & & \\
\hline rarely / rzadko & $244(38.36)$ & $54(26.73)$ & & $142(38.80)$ & $66(43.42)$ & & \\
\hline often / często & $240(37.74)$ & $98(48.51)$ & & $96(26.23)$ & $44(28.95)$ & & \\
\hline regularly / stale & $52(8.18)$ & $36(17.82)$ & 0.10 & $30(8.20)$ & $18(11.84)$ & 0.02 & 0.01 \\
\hline \multicolumn{8}{|l|}{ Parental absence / Nieobecność rodziców } \\
\hline almost never / prawie nigdy & $84(13.21)$ & $20(9.90)$ & & $78(21.31)$ & $34(22.37)$ & & \\
\hline rarely / rzadko & $316(49.69)$ & $82(40.59)$ & & $162(44.26)$ & $64(42.11)$ & & \\
\hline often / często & $182(28.62)$ & $72(35.64)$ & & $106(28.96)$ & $44(28.95)$ & & \\
\hline regularly / stale & $54(8.49)$ & $28(13.86)$ & 0.09 & $20(5.46)$ & $10(6.58)$ & 0.95 & 0.10 \\
\hline \multicolumn{8}{|l|}{ Parental care / Opieka rodzicielska } \\
\hline they do not care at all / żadna & $4(0.63)$ & $4(1.98)$ & & $6(1.64)$ & $0(0.00)$ & & \\
\hline they do not care enough / niewystarczająca & $24(3.77)$ & $14(6.93)$ & & $16(4.37)$ & $8(5.26)$ & & \\
\hline relatively concerned / stosunkowo dobra & $78(12.26)$ & $34(16.83)$ & & $86(23.50)$ & $46(30.26)$ & & \\
\hline highly / bardzo dobra & $530(83.33)$ & $150(74.26)$ & 0.19 & $258(70.49)$ & $98(64.47)$ & * & 0.01 \\
\hline \multicolumn{8}{|l|}{$\begin{array}{l}\text { Parental questioning / Nadmierne } \\
\text { zainteresowanie rodziców }\end{array}$} \\
\hline almost never / prawie nigdy & $30(4.72)$ & $12(5.94)$ & & $18(4.92)$ & $4(2.63)$ & & \\
\hline rarely / rzadko & $40(6.29)$ & $18(8.91)$ & & $30(8.20)$ & $12(7.89)$ & & \\
\hline often / często & $116(18.24)$ & $30(14.85)$ & & $128(34.97)$ & $42(27.63)$ & & \\
\hline regularly / stale & $450(70.75)$ & $142(70.30)$ & 0.62 & $190(51.91)$ & $94(61.84)$ & 0.14 & 0.00 \\
\hline
\end{tabular}

Abbreviations as in Table 2 / Skróty jak w tabeli 2.

Table 6. Smoking incidence among girls and boys - forward conditional logistic regression

Tabela 6. Palenie papierosów wśród dziewcząt i chłopców - analiza regresji logistycznej metodą krokową

\begin{tabular}{|c|c|c|c|c|}
\hline \multirow[t]{2}{*}{$\begin{array}{l}\text { Condition } \\
\text { Warunek }\end{array}$} & \multicolumn{2}{|c|}{$\begin{array}{c}\text { Girls } \\
\text { Dziewczęta } \\
(\mathrm{N}=636) \\
{[\mathrm{n}(\%)]}\end{array}$} & \multicolumn{2}{|c|}{$\begin{array}{c}\text { Boys } \\
\text { Chłopcy } \\
(\mathrm{N}=366) \\
{[\mathrm{n}(\%)]}\end{array}$} \\
\hline & $\mathrm{OR}(\mathrm{p})$ & $95 \% \mathrm{CI}$ & OR $(\mathrm{p})$ & $95 \% \mathrm{CI}$ \\
\hline \multicolumn{5}{|l|}{ Step 1 / Krok 1} \\
\hline constant / stała & $0.053(0.01)$ & & $0.194(0.01)$ & \\
\hline school absence / nieobecność w szkole & & & $1.623(0.01)$ & $1.283-2.054$ \\
\hline familial (parental) conflicts / konflikty z rodzicami & $2.391(0.01)$ & $1.732-3.302$ & & \\
\hline \multicolumn{5}{|l|}{ Step 2 / Krok 2} \\
\hline constant / stała & $0.020(0.01)$ & & $0.114(0.01)$ & \\
\hline school absence / nieobecność w szkole & $1.719(0.01)$ & $1.258-2.348$ & $1.474(0.03)$ & $1.025-2.120$ \\
\hline unexcused school absence / nieusprawiedliwiona nieobecność w szkole & & & $1.396(0.03)$ & $1.063-2.243$ \\
\hline familial (parental) conflicts / konflikty z rodzicami & $2.097(0.01)$ & $1.501-2.931$ & & \\
\hline
\end{tabular}


Table 6. Smoking incidence among girls and boys - forward conditional logistic regression - cont.

Tabela 6. Palenie papierosów wśród dziewcząt i chłopców - analiza regresji logistycznej metodą krokową - cd.

\begin{tabular}{|c|c|c|c|c|}
\hline \multirow[t]{2}{*}{$\begin{array}{l}\text { Condition } \\
\text { Warunek }\end{array}$} & \multicolumn{2}{|c|}{$\begin{array}{c}\text { Girls } \\
\text { Dziewczęta } \\
(\mathrm{N}=636) \\
{[\mathrm{n}(\%)]}\end{array}$} & \multicolumn{2}{|c|}{$\begin{array}{c}\text { Boys } \\
\text { Chłopcy } \\
(\mathrm{N}=366) \\
{[\mathrm{n}(\%)]}\end{array}$} \\
\hline & OR $(\mathrm{p})$ & $95 \% \mathrm{CI}$ & OR $(\mathrm{p})$ & $95 \% \mathrm{CI}$ \\
\hline \multicolumn{5}{|l|}{ Step 3 / Krok 3} \\
\hline constant / stała & $0.014(0.01)$ & & $0.026(0.01)$ & \\
\hline school absence / nieobecność w szkole & $1.596(0.01)$ & $1.161-2.193$ & $1.544(0.03)$ & $1.063-2.243$ \\
\hline unexcused school absence / nieusprawiedliwiona nieobecność w szkole & & & $1.360(0.04)$ & $1.029-1.796$ \\
\hline scholastic achievement / postępy w nauce & $1.484(0.01)$ & $1.105-1.993$ & & \\
\hline parental questioning / nadmierne zainteresowanie rodziców & & & $1.530(0.04)$ & $1.020-2.295$ \\
\hline familial (parental) conflicts / konflikty z rodzicami & $2.029(0.01)$ & $1.447-2.847$ & & \\
\hline \multicolumn{5}{|l|}{ Step 4 / Krok 4} \\
\hline constant / stała & $0.104(0.04)$ & & & \\
\hline school absence / nieobecność w szkole & $1.565(0.01)$ & $1.137-2.155$ & & \\
\hline scholastic achievement / postępy w nauce & $1.467(0.04)$ & $1.089-1.977$ & & \\
\hline familial (parental) conflicts / konflikty z rodzicami & $1.979(0.01)$ & $0.377-0.962$ & & \\
\hline familial (parental) care / opieka rozdzicielska & $0.602(0.04)$ & $0.377-0.962$ & & \\
\hline
\end{tabular}

OR - odds ratio / iloraz szans, CI - confidence interval / przedział ufności.

the strong relationships between cigarette smoking and educational variables and between cigarette smoking and familial monitoring.

To the best of our knowledge, only 1 recent study has investigated the rate of smoking among adolescents in Kosovo [2]. In this study, the authors have reported a prevalence of smoking in the town of Gilan (34.8\% and $37.4 \%$ for girls and boys, respectively) that is consistent with our findings ( $31.8 \%$ and $41.5 \%$ for girls and boys, respectively). We can therefore acknowledge that high rates of cigarette smoking among adolescents is likely one of the most important public health issues in Kosovo. Previous studies conducted across the former Yugoslav territory have shown that a high rate of cigarette smoking is not a problem unique to Kosovo. Accordingly, a study that investigated Croatian adolescents has found an even higher smoking rate: $57 \%$ of males and $51 \%$ of females were smokers, including $31 \%$ and $36 \%$ considered to be daily smokers, respectively [3]. A similar prevalence has recently been reported for Bosnian-Herzegovinian adolescents ( $55 \%$ of males and $40 \%$ of females were smokers, including $35 \%$ and $15 \%$ considered to be daily smokers, respectively) $[4,17]$. Therefore, national policies and market regulations that are regularly considered the most important factors related to smoking prevalence are likely not to be as important as expected.

For example, Croatian legislation regarding cigarette marketing is more rigid than the legislation in Bosnia and Herzegovina and Kosovo. However, this discrepancy has not been reflected in differences in smoking rates among adolescents in these 3 countries. In fact, the prevalence of daily smoking has been highest among Croatian adolescents $(13 \%, 25 \%$, and $33 \%$ for Kosovo, Bosnia and Herzegovina, and Croatia, respectively). Therefore, other issues should be considered in the course of assessments of the factors influencing adolescents' smoking. The national economic situation and the effect on discretionary income is likely to be a significant factor. Accordingly, current reports show a clear difference between former Yugoslav republics in per capita income (USD 13 105; USD 3517; and USD 2813 for Croatia, Bosnia and Herzegovina and Kosovo, respectively) [18].

Our results show similar predictors of smoking in both genders. These findings support the development of an effective national campaign against smoking in Kosovo. Whereas preventive efforts in other countries have to be somewhat gender-specific, our findings support the use of preventive actions that will be equally applicable to boys and girls. One could argue that 
our findings are influenced by our sampling of subjects from the capital of Kosovo and related sociodemographic structures. However, the smoking rate found in our sample has been consistent with the rates reported previously for adolescents in other towns in Kosovo (i.e., Gjilan), which supports our findings.

Sports and physical exercise provide numerous health and social benefits for young people [19]. However, sports participation is often uncritically suggested as a protective factor against substance misuse. One frequent explanation is that organised activities such as sports may be protective by facilitating prosocial group membership, while other explanations highlight the incompatibility of physical exercise and cigarettes due to the known adverse impact of cigarette smoking upon fitness capacities [16]. However, investigations have provided inconsistent findings about the relationship between sports participation and smoking among adolescents $[4,7,8]$.

In this study, the relationship between sports participation and cigarette smoking among adolescents in Kosovo has been weak. Briefly, sport-related factors have not been found to be significant predictors in logistic regressions. Meanwhile, participation in team sports has been the only sport-related factor that has been univariately associated with smoking among males. However, this finding has been highly specific. Accordingly, those boys who once practised team sports and subsequently quit are more likely to be smokers. This finding supports the findings of recent studies which have recognised the differential influence of team sports and individual sports on substance use and misuse in Croatian and Bosnian and Herzegovinian adolescents $[3,4]$.

The results of our study directly support previous studies, in which conflict with parents (i.e., family members) was found to be a strong risk factor for adolescent substance misuse, including cigarette smoking [20,21]. It is logical that more frequent parent-child conflicts put children in out-of-home situations, in which they are in contact with peers who misuse substances and are consequently more vulnerable to substance misuse. However, it seems that conflicts with parents account for a stronger predictive factor for cigarette smoking in the case of girls than boys.

More precisely, out of 4 parental monitoring variables examined among boys, only self-reported parental questioning has turned out to be an influential factor (i.e., those boys who are more often questioned about their personal life, school, and friends are more likely to be smokers). Self-reported conflicts with parents account for a significant predictor of smoking solely among girls.
The question that arises is whether smoking influences "questioning" (for boys) and "conflicts" (for girls) or whether parental pressure (through "questioning" and "conflicts") result in out-of-home situations and consequently lead to smoking.

A strong association has been found between scholastic variables and smoking, and a higher rate of smoking has been evidenced among those subjects who struggle educationally. These results have been expected because nearly all studies performed to date had reported a negative association between educational achievement and smoking $[4,22,23]$. In our study, this phenomenon has been more obvious among girls than among boys, likely due to better scholastic achievements among girls.

However, similarly to the previously discussed relationship between familial monitoring and smoking, it is unclear whether educational failure leads to smoking or whether those who smoke have started to fail themselves educationally. There is theoretical support for both explanations; whereas the former mechanism can be explained by social-peer theory, the latter mechanism is supported by known physiological mechanisms and the adverse impact of smoking upon cognitive ability and memory $[4,22]$. Currently, we can neither support nor deny either of these theories because a longitudinal (i.e., follow-up) study, that will clearly define the cause-effect relationships between the studied variables, is needed.

\section{Limitations}

This has been a cross-sectional study that has provided insights into the associations between the studied variables but causality has not been investigated. However, this is one of the 1st studies to investigate the factors related to cigarette smoking in Kosovo, and we are aware that an interventional approach will be needed in future studies. Finally, we have surveyed children in only 1 religious group in Kosovo (ethnic Albanians who identified themselves to be Islamic) but we have done so intentionally because previous studies had demonstrated the need to be alert to religion and ethnicity when investigating the problem of substance use among adolescents.

\section{CONCLUSIONS}

The incidence of smoking among adolescents in Kosovo is high but within expected values. Smoking is almost certainly culturally influenced and boosted by the relatively low price of tobacco and the availability of cigarettes across the territory of former Yugoslavia. 
Given the lack of a national strategy for substance misuse prevention, it is reasonable to suggest that the data reported herein should be used in preventative efforts aimed at reducing the prevalence of smoking in Kosovo. More specifically, a higher risk of smoking is expected among girls who report themselves to be in a higher conflict with their parents and at the same time perceive low levels of parental care about them and their personal life. These girls are also at a higher risk of poor academic achievements. Among boys, smoking is more frequent for those boys who perceive stronger parental questioning, who are absent from school more often and who consequently have more unexcused absences.

The sport-related factors included in this study have not turned out to be related to smoking among girls. However, there has been evidence that discontinuation of participation in team sports is a risk factor for smoking among boys.

\section{ACKNOWLEDGMENTS}

Authors gratefully acknowledge the help of school authorities from Pristina, Kosovo. Special thanks goes to all participants and their parents.

\section{REFERENCES}

1. Vink JM, Boomsma DI. Interplay between heritability of smoking and environmental conditions? A comparison of two birth cohorts. BMC Public Health. 2011;11:316, http://dx.doi.org/10.1186/1471-2458-11-316.

2. Carkaxhiu L, Huseyin K, Berisha M, Botica MV. Problem of substance misuse and lack of national strategy in Kosovo. Cent Eur J Public Health. 2011;19(2):108-14.

3. Modric T, Zenic N, Sekulic D. Substance use and misuse among 17- to 18-year-old Croatian adolescents: Correlation with scholastic variables and sport factors. Subst Use Misuse. 2011;46(10):1328-34, http://dx.doi.org/10.3109/ 10826084.2011.579677.

4. Sekulic D, Ostojic M, Ostojic Z, Hajdarevic B, Ostojic L. Substance abuse prevalence and its relation to scholastic achievement and sport factors: An analysis among adolescents of the Herzegovina-Neretva Canton in Bosnia and Herzegovina. BMC Public Health. 2012;12(1):274, http:// dx.doi.org/10.1186/1471-2458-12-274.

5. Kaleta D, Korytkowski P, Makowiec-Dąbrowska T. [Cigarette smoking among economically active population]. Med Pr. 2013;64(3):359-71. Polish.

6. Vajngerl B, Videmsek M, Karpljuk D, Stihec J. Analysis of sport activity and smoking of 15-year- old students and their parents. Kinesiol Slov. 2008; 14(1):56-67.

7. Rodriguez Garcia PL, Lopez Villalba FJ, Lopez Minarro PA, Garcia Canto E. [Relationship between tobacco consumption and physical exercise in adolescents. Differences between genders]. Adicciones. 2013;25(1):29-36. Spanish. 8. Guo H, Reeder AI, McGee R, Darling H. Adolescents leisure activities, parental monitoring and cigarette smoking - A cross-sectional study. Subst Abuse Treat Prev Policy. 2011;6:12, http://dx.doi.org/10.1186/1747-597X-6-12.

9. Cavar M, Sekulic D, Culjak Z. Complex interaction of religiousness with other factors in relation to substance use and misuse among female athletes. J Relig Health. 2012;51(2):381-9, http://dx.doi.org/10.1007/s10943-0109360-9.

10. Sanchez ZM, Opaleye ES, Martins SS, Ahluwalia JS, Noto AR. Adolescent gender differences in the determinants of tobacco smoking: A cross sectional survey among high school students in Sao Paulo. BMC Public Health. 2010;10:748, http://dx.doi.org/10.1186/1471-2458-10-748.

11. Soweid RAA, Khawaja M, Salem MT. Religious identity and smoking behavior among adolescents: Evidence from entering students at the American University of Beirut. Health Commun. 2004;16(1):47-62, http://dx.doi. org/10.1207/S15327027HC1601_4.

12. Zenic N, Stipic M, Sekulic D. Religiousness as a factor of hesitation against doping behavior in college-age athletes. J Relig Health. 2013;52(2):386-96, http://dx.doi.org/ 10.1007/s10943-011-9480-x.

13. Branstetter SA, Furman W. Buffering effect of parental monitoring knowledge and parent-adolescent relationships on consequences of adolescent substance use. J Child Fam Stud. 2013;22(2):192-8, http://dx.doi.org/ 10.1007/s10826-012-9568-2.

14. Mason WA, Haggerty KP, Fleming AP, Casey-Goldstein M. Family intervention to prevent depression and substance use among adolescents of depressed parents. J Child Fam Stud. 2012;21(6):891-905, http://dx.doi.org/10.1007/ s10826-011-9549-x.

15. Mandic GF, Peric M, Krzelj L, Stankovic S, Zenic N. Sports nutrition and doping factors in synchronized swimming: Parallel analysis among athletes and coaches. J Sport Sci Med. 2013;12(4):753-60.

16. Sekulic D, Milanovic I, Bok D, Jukic I, Matika D. Substance use and misuse in the Croatian Army Special Forces: Prevalence and influencing factors. Int J Occup Med Environ Health. 2014;27(1):123-31, http://dx.doi.org/10.2478/ s13382-014-0241-4.

17. Sekulic D, Ostojic M, Vasilj M, Coric S, Zenic N. Genderspecific predictors of cigarette smoking in adolescents: 
An analysis of sport participation, parental factors and religiosity as protective/risk factors. J Subst Use. 2014;19(1-2): 89-94, http://dx.doi.org/10.3109/14659891.2012.734544.

18. Per capita GDP at current prices - US dollars. The United Nations Statistics Division [cited 2014 Oct 28]. Available from: https://data.un.org/Data.aspx?d=SNAAMA\&f=grI D\%3A101\%3BcurrID\%3AUSD\%3BpcFlag\%3A1.

19. Bradley J, Keane F, Crawford S. School sport and academic achievement. J Sch Health. 2013;83(1):8-13, http://dx.doi. org/10.1111/j.1746-1561.2012.00741.x.

20. Jeganathan PD, Hairi NN, Al Sadat N, Chinna K. Smoking stage relations to peer, school and parental factors among secondary school students in Kinta, Perak. Asian
Pac J Cancer Prev. 2013;14(6):3483-9, http://dx.doi.org/ 10.7314/APJCP.2013.14.6.3483.

21. Bobakova D, Geckova AM, Klein D, Reijneveld SA, van Dijk JP. Protective factors of substance use in youth subcultures. Addict Behav. 2012;37(9):1063-7, http://dx. doi.org/10.1016/j.addbeh.2012.04.014.

22. So W-Y. Association between cigarette consumption and academic achievement in Korean adolescents. Addiction Res Theory. 2014(22):424-30, http://dx.doi.org/10.3109/ 16066359.2013 .874417$.

23. Chung SS, Joung KH. Risk factors for smoking behaviors among adolescents. J Sch Nurs. 2014;30(4):262-71, http:// dx.doi.org/10.1177/1059840513505222.

This work is published in Open Acess and licensed under a Creative Commons Attribution-NonCommercial 3.0 Unported License / Ten utwór jest dostępny w modelu open access na licencji Creative Commons Uznanie autorstwa - Użycie niekomercyjne 3.0 Unported - https://creativecommons.org/licenses/ by-nc/3.0/deed.en. 